\title{
Indicadores de sustentabilidade em agroecossistemas de urucuzeiro e sua relação com os visitantes florais
}

A biodiversidade através de seus atributos funcionais tem desempenhado um papel fundamental na sustentabilidade e no equilíbrio dos agroecossistemas, contribuindo para a geração de serviços ecossistêmicos. Assim, objetivou-se determinar a sustentabilidade de cultivos de urucuzeiro e sua relação com a riqueza, diversidade e abundância de visitantes florais. Os dados foram coletados em 15 (quinze) cultivos de urucuzeiro, localizados no Território Vale do Guaporé, região sudoeste do Estado de Rondônia. Para avaliar sustentabilidade do cultivo, fo empregada metodologia de avaliação agroecológica de sustentabilidade onde o indicador é composto de grupos de indicadores de qualidade do solo e de saúde dos cultivos. 0 estudo dos visitantes florais do urucuzeiro foi realizado por meio de observação, coleta ativa e coleta passiva e os organismos coletados após a classificação taxonômica, foram reunidos em grupos
funcionais. A média obtida nos quinze agroecossistemas para a qualidade do solo foi de 9,1 e para a saúde dos cultivos foi de 7,7 , os indicadores apontaram que os agroecossistemas de urucuzeiro são sustentáveis por apresentarem valores próximos ao máximo. Foram observados visitando as flores do urucuzeiro um grupo diverso de artrópodes, da classe Arachnida e da classe Insecta, sendo a ordem Hymenoptera a mais visualizada e presente nos agroecossistemas. As abelhas das famílias Andrenidae, Apidae e Halictidae, foram as mais ocorrentes nas áreas de estudo, na coleta ativa foram capturadas 39 espécies (morfotipos) de abelhas, sendo 16 espécies (morfotipos) de abelhas de maior tamanho corporal e 23 espécies (morfotipos) de meno tamanho corporal, sendo que as de pequeno porte, 18 apresentavam capacidade vibratória e 5 delas não apresentavam comportamento vibratório ao coletar o pólen. Os polinizadore efetivos e ocasionais ocorreram em todos os agroecossistemas estudados. Na coleta passiva, a ordem Hymenoptera apresentou uma riqueza de 51 morfotipos, com abundância de 737 indivíduos, distribuídos nas famílias Apidae, Halictidae, Formicidae e Vespidae. A riqueza, diversidade e abundância de artrópodes não manteve relação direta com os indicadores de sustentabilidade, na medida em que os indicadores se aproximavam ao valor máximo de sustentabilidade para os agroecossistemas, a riqueza, a diversidade e abundância não mantiveram a tendência. Indicando que outras variáveis não mensuradas devem ser analisadas em conjunto com atributos do solo e saúde dos cultivos para garantir os serviços ecossistêmicos providos por esses grupos funcionais.

Palavras-chave: Serviços ecossistêmicos; Grupos funcionais; Qualidade do solo; Polinização.

\section{Sustainability indicators in agroecosystems annatto and its relationship with florais visitors}

\begin{abstract}
Biodiversity through its functional attributes has played a fundamental role in the sustainability and balance of agroecosystems, contributing to the generation of ecosystem services. Thus, the objective was to determine the sustainability of annatto tree crops and their relation with the richness, diversity and abundance of floral visitors. The data were collected in 15 (fifteen) annatto crops, located in the Vale do Guaporé Territory, southwest region of the State of Rondônia. In order to evaluate the sustainability of the crop, an agroecological sustainability assessment methodology was used where the indicator is composed of groups of indicators of soil quality and crop health. The study of the floral visitors of annatto was accomplished by means of observation, active collection and passive collection and the organisms collected after the taxonomic classification were collected in functional groups. The average obtained in the fifteen agroecosystems for the soil quality was 9.1 and for the health of the crops was 7.7 , the indicators pointed out that the agroecosystems of annatto are sustainable because they present visualized and present in agroecosystems. The bees of the Andrenidae, Apidae and Halictidae families were the most frequent in the study areas, 39 species (morphotypes) of bees were captured in the active collection, being 16 species (morphotypes) of bees with larger body size and 23 species (morphotypes) of smaller body size, and those of small size, 18 presented vibratory capacity and 5 of them did not present vibratory behavior when collecting the pollen. Effective and occasional pollinators occurred in all studied agroecosystems. In the passive collection, the order Hymenoptera presented a richness of 51 morphotypes, with abundance of 737 individuals, distributed in the families Apidae, Halictidae, Formicidae and Vespidae. The wealth, diversity and abundance of hymenoptera are not directly related to sustainability indicators.
\end{abstract}

Keywords: Ecosystem services; Functional groups; Soil quality; Pollination.

Topic: Conservação da Biodiversidade

Reviewed anonymously in the process of blind peer.

Sérgio Aparecido Seixas da Silva (iD

Universidade Federal de Rondônia, Brasil

http://lattes.cnpq.br/1316242208343441

http://orcid.org/0000-0001-5640-7051

sergioseixassilva@gmail.com

Suellen Fernanda Mangueira Rodrigues

Universidade Federal de Rondônia, Brasil

http://lattes.cnpq.br/7505779810779510

sfernandamangueira@gmail.com

Anna Frida Hatsue Modro (iD

Universidade Federal de Rondônia, Brasil

http://lattes.cnpq.br/9474501340526969

http://orcid.org/0000-0002-6201-960X

anna.frida@unir.br

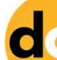

DOI: 10.6008/CBPC2179-6858.2019.001.0004
Received: 02/12/2018

Approved: 26/01/2019

Eduardo Cândido Franco Rosell

Universidade Federal de Rondônia, Brasil http://lattes.cnpq.br/1721533687890440

edyrosell@unir.br

Emanuel Fernando Maia de Souza ii

Universidade Federal de Rondônia, Brasil

http://lattes.cnpq.br/4742387914768064

http://orcid.org/0000-0002-5493-2183

emanuel@unir.br

Referencing this:

SILVA, S. A. S.; RODRIGUES, S. F. M.; MODRO, A. F. H.; ROSELL, E. C. F.; SOUZA, E. F. M.. Indicadores de sustentabilidade em agroecossistemas de urucuzeiro e sua relação com os visitantes florais. Revista Ibero Americana de Ciências Ambientais, v.10, n.1, p.29-45, 2019. DOI: http://doi.org/10.6008/CBPC2179-6858.2019.001.0004 


\section{INTRODUÇÃO}

Os serviços ecossistêmicos são processos naturais biológicos, geológicos e químicos que ocorrem em ecossistemas saudáveis (KEARNS et al., 1998). Que colaboram direta e indiretamente com a sobrevivência das espécies no planeta Terra e têm a capacidade de prover bens e serviços que satisfazem as necessidades humanas (COSTANZA et al., 1997; DAILY, 1997; GROOT et al., 2002; ALHO, 2012). A biodiversidade através de seus atributos, tais como composição, riqueza, abundância, influência diretamente ou indiretamente os serviços ecossistêmicos por afetar a magnitude, o ritmo, a continuidade temporal pelo qual a energia e os materiais são distribuídos ao longo dos ecossistemas (DÍAZ et al., 2006).

Sendo assim, a perda de biodiversidade gera extinção de organismos que tem uma determinada função ecossistêmica, sem os quais, outros organismos serão afetados, portanto todo o ecossistema de um determinado local será alterado e os serviços ecossistêmicos serão modificados. O que torna necessária a manutenção da biodiversidade funcional, a qual engloba o conjunto de características funcionais provenientes de espécies individuais ou de grupos de espécies para sustentar as funções e os processos ecossistêmicos (SANDIFER et al., 2015).

A sustentabilidade da paisagem rural garante a permanência e a continuidade dos serviços ecossistêmicos, influenciando diretamente na qualidade do solo, na saúde dos cultivos e na diversificação da diversidade biológica funcional (MACHADO et al., 2006). Entre os principais serviços a polinização merece destaque, pois é um dos componentes essenciais para a manutenção e o bom funcionamento dos ecossistemas (POTTS et al., 2010), sendo os insetos o grupo de polinizadores mais expressivo, pois participam da polinização de um grande número de espécies de plantas, tanto em ambientes não antropizados quanto em cultivos agrícolas (KEARNS et al., 1998). A produção de frutas, hortaliças e sementes de $75 \%$ das principais culturas alimentares globais depende da polinização animal, enquanto $25 \%$ das culturas não dependem deste serviço (KLEIN et al., 2006). Cerca de aproximadamente $73 \%$ das espécies vegetais cultivadas no mundo são polinizadas por alguma espécie de abelha, $19 \%$ por moscas, $6,5 \%$ por morcegos, $5 \%$ por vespas, 5\% por besouros, $4 \%$ por pássaros e $4 \%$ por borboletas e mariposas (FAO, 2004).

Das 141 culturas analisadas no Brasil, verificou-se que 85 delas dependem de polinizadores, a contribuição econômica dos polinizadores totaliza quase 30\% (aproximadamente US\$12 bilhões) do valor total da produção agrícola anual das culturas dependentes (totalizando quase US\$45 bilhões) (GIANNINI et al., 2015). Por outro lado, as abelhas representam, o grupo mais importante de polinizadores (BAWA et al., 1985) e são dependentes das flores para obtenção de recursos alimentares (néctar, pólen, óleo) (CORBET et al., 1991; SANTOS et al., 2006; YAMAMOTO et al., 2012).

Nas últimas décadas, a intensificação da agricultura e pecuária em países em desenvolvimento provocou grandes alterações ambientais, tais como conversão de áreas nativas em áreas de cultivo e criações. O que compromete as comunidades de abelhas selvagens (KLEIN et al., 2007). Além da diminuição de espécies, essas alterações, podem levar a limitações das atividades dos polinizadores e afetar a reprodução de plantas com flores (AGUILAR et al., 2006), levando à geração de uma série de desserviços 
ambientais e com consequente perda dos serviços ecossistêmicos (ZHANG et al., 2007). A gestão e o manejo dos serviços de polinização natural podem ajudar a sustentar a diversidade e a produção das culturas (KLEIN et al., 2007).

As causas e interrupções nos sistemas de polinização pode estar relacionada a diversos aspectos entre eles, a diminuição e fragmentação de habitats, mudanças no uso da terra, uso de agrotóxicos, como pesticidas e herbicidas, patógenos, introdução de espécies invasoras e mudanças climáticas (KEARNS et al., 1998; KEVAN et al., 2003; POTTS et al., 2010; GOULSON et al., 2015). Causando o declínio de polinizadores e resultando na crise da polinização, que está associada às atividades humanas (KEARNS et al., 1998; KEVAN et al., 2001; KEVAN et al., 2003).

É importante ressaltar que a importância econômica do serviço de polinização vem sendo relatada por vários estudos (COSTANZA et al., 1997; KLEIN et al., 2007; GALLAl et al., 2009; BAUER et al., 2010; POTTS et al., 2010; GIANNINI et al., 2015). E existem muitas informações sobre polinização realizada por abelhas em um grande número de culturas de importância econômica no Brasil (D’AVILA et al., 2005).

O urucuzeiro (Bixa orellana L.) é um arbusto perene da família Bixaceae, originário da parte norte da América do Sul, especificamente na região Amazônica (SANDY-CUEN et al., 2003; CLEMENT et al., 2010). Amplamente distribuído na região Amazônica até a Bahia, geralmente próximos de rios (LORENZI, 2002). Ocorre também em diversos países da América do Sul, América Central até o México. O urucuzeiro (Bixa orellana L.) é uma planta predominantemente xenógama, polinizada por uma guilda de visitantes florais (MESQUITA, 2008), principalmente por abelhas (ALMEIDA et al., 1992; COSTA, 2005; COSTA et al., 2008; BONFIM et al., 2015; ROCHA et al., 2017).

Vários esforços têm sido empreendidos na tentativa de buscar manejos e tecnologias que contribuam para a sustentabilidade dos agroecossistemas, e considerando que o produto final do cultivo do urucuzeiro é o grão oriundo do fruto e que sem os serviços ecossistêmicos de polinização não é possível produzir seus, torna-se notável a importância e a necessidade de compreender o nível de sustentabilidade nos agroecossistemas e a fauna de artrópodes visitantes florais presente. Onde o cultivo depende de agentes polinizadores bióticos para frutificar satisfatoriamente. Sendo assim, esse trabalho objetivou determinar a sustentabilidade de cultivos de urucuzeiro (Bixa orellana L.) e sua relação com a riqueza, diversidade e abundância da fauna de visitantes florais.

\section{MATERIAIS E MÉTODOS}

\section{Caracterização da área de estudo}

As áreas de cultivo do urucuzeiro foram selecionadas a partir da indicação da Cooperativa de Produtores Rurais Vale do Guaporé de Seringueiras - COOPERVAGS, os quais indicaram 15 agroecossistemas, distribuídos na zona rural dos municípios de Seringueiras e São Miguel do Guaporé, Território Vale do Guaporé, região localizada na porção sudoeste do Estado de Rondônia, Amazônia Ocidental (Figura 1). Os 15 agroecossistemas foram classificados por letras (A, B, C, D, E, F, G, H, I, J, K, L, M, N e O). 


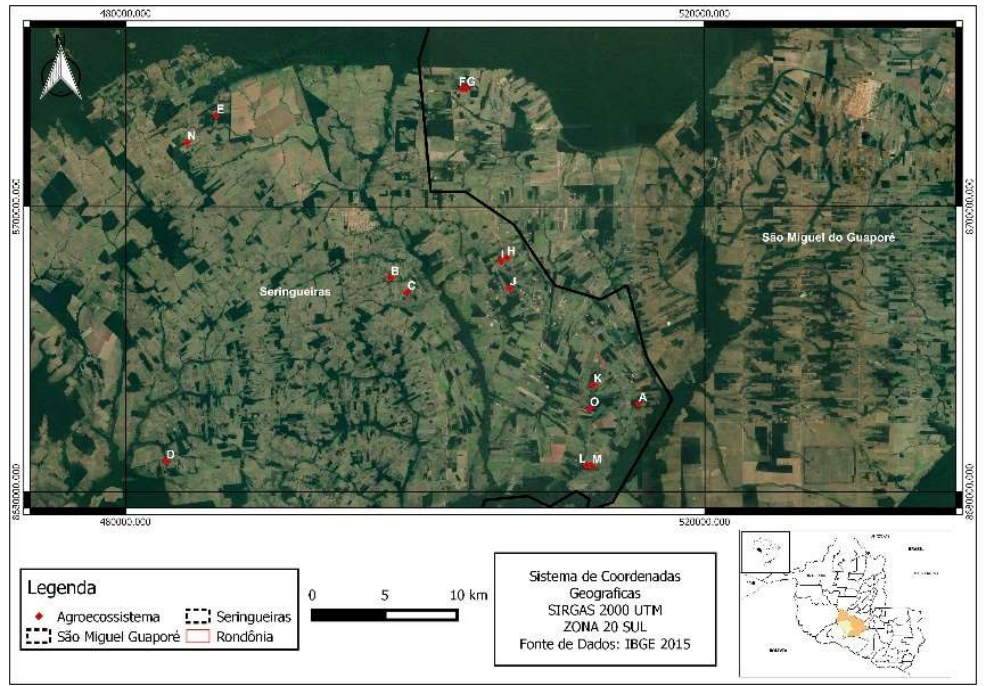

Figura 1: Distribuição dos agroecossistemas de urucuzeiro nos municípios de Seringueiras e São Miguel do Guaporé utilizados como áreas de levantamento de visitantes florais, cujas coletas foram realizadas em 2018, Amazônia Ocidental, Brasil.

O clima predominante na região é do tipo 'Am' - Clima Tropical de monção, com média climatológica da temperatura anual entre 24 e 26 ㄷ, e precipitação média entre 1900 a 2500 mm/ano (ALVARES et al., 2014). Com duas estações marcadamente distintas: seca (junho a agosto) e chuvosa (outubro a abril), maio e setembro são meses de transição. $O$ período chuvoso concentra cerca de $70 \%$ da precipitação anual, sendo o primeiro trimestre do ano o de maior precipitação. A média da precipitação para os meses mais secos do ano é inferior a $50 \mathrm{~mm} / \mathrm{mês}$ (RONDÔNIA, 2012).

A vegetação predominante, caracteriza-se por Floresta Ombrófila Aberta Submontana (IBGE, 2012). Os solos que prevalecem na região são latossolos amarelos distróficos e latossolos vermelho-amarelos distróficos com argissolos vermelho-amarelos distróficos, ambos típicos, com relevo plano e suave ondulado, com altitude variando entre 100 e 200 metros (SANTOS et al., 2011; IBGE, 2015).

\section{Análise da sustentabilidade}

Para avaliar a qualidade do solo e a saúde dos cultivos em agroecossistemas de urucuzeiro, foram utilizados os indicadores de sustentabilidade propostos por Altieri et al. (2002) e Nicholls et al. (2004). Os indicadores foram escolhidos por possuírem princípios fáceis de serem aplicados, analisados e entendidos pelos agricultores; rápidos e de baixo custo na aplicação e no monitoramento; e claramente definidos. A coleta de dados dos indicadores foi realizada no mês de janeiro de 2018, entre os dias 09 e 26.

Valores entre um e dez foram atribuídos para cada indicador, em cada sistema, foram analisados três pontos no sentido diagonal do cultivo, respeitando-se $10 \mathrm{~m}$ de borda em cada extremo. Os valores próximos de 1 estão atrelados a manejo não adequado ou não desejável, sendo os valores próximos de 5 como medianos ou moderados e acima de 5 e próximos de 10 como ideais ou desejáveis. Notas abaixo de 5 são consideradas abaixo do limite mínimo de sustentabilidade. As médias dos indicadores qualidade do solo e saúde do cultivo foram utilizadas para a construção do indicador geral. 


\section{Amostragem, observação e coleta da fauna de visitantes florais do urucuzeiro}

As observações foram realizadas um dia para cada agroecossistema estudado, durante o período de floração do cultivo para a região, entre os meses de fevereiro e maio de 2018 , em dois períodos do dia, sendo das 06:00h às 11h:30min e das 13:00h às 18h:10min, totalizando aproximadamente 160 horas de observação. Onde foi percorrido um transecto em formato de X, de forma lenta e contínua, observando-se a ocorrência, o comportamento da fauna de visitantes florais, informações sobre o horário de visitação, dados meteorológicos foram anotados. Quando possível foi realizada a coleta ativa e registros fotográficos de visitantes florais para auxiliar na identificação. Nas coletas ativas foi utilizado o auxílio de puçá (rede entomológica), ou manualmente, colocando os artrópodes coletados em um frasco letal contendo formol para sacrificá-los.

Em cada agroecossistema também foi realizado um estudo complementar por meio de coleta passiva, com o uso de armadilhas para capturar a fauna de artrópodes associada ao urucuzeiro. As armadilhas de interceptação de voo foram confeccionadas com garrafas PET de 2 litros, transparentes, com três aberturas de $5 \times 5 \mathrm{~cm}$, com distância de $6 \mathrm{~cm}$ de uma para outra, instaladas a 1,5 m do solo (LIMA FILHO et al., 2014). As armadilhas foram instaladas na diagonal do cultivo, onde para cada área foram distribuídas três armadilhas de interceptação de voo, iscadas com suco de abacaxi, ficando expostas no campo por 72 horas. Os sucos-iscas foram preparados com $100 \mathrm{~mL}$ de suco da fruta e $250 \mathrm{~mL}$ de água, totalizando $350 \mathrm{~mL}$. Em todas as iscas, foram acrescentadas 3 gotas de detergente neutro (LIMA FILHO et al., 2013).

A fauna coletada foi encaminhada ao LaHorTa - Laboratório de Horticultura Tropical e Apicultura da Universidade Federal de Rondônia (UNIR), Campus Rolim de Moura, onde foi realizado o procedimento de armazenamento e conservação adequado à cada grupo de animal. A identificação dos morfotipos foi feita com o auxílio da chave de identificação proposta para abelhas (SILVEIRA et al., 2002), vespas (CARPENTER et al., 2001) e formigas (BACCARO, 2006).

Os organismos coletados da ordem Hymenoptera após a classificação taxonômica, foram reunidos nos seguintes grupos funcionais: as espécies de abelhas que possuem grande tamanho corporal, representadas por indivíduos com mais de $14 \mathrm{~mm}$ de comprimento e $6 \mathrm{~mm}$ de largura (VIANA et al., 2005), foram classificadas como polinizadores efetivos (P.E) (ALMEIDA et al., 1992). As abelhas de menor tamanho corporal, inferior a $14 \mathrm{~mm}$ de comprimento e $6 \mathrm{~mm}$ de largura (VIANA et al., 2005), foram classificadas como polinizadores ocasionais (P.O) (ALMEIDA et al., 1992). O comportamento das abelhas foi separado em: com vibração (C.V) e sem vibração (S.V), os outros himenópteros (vespas - Vespidae; formigas - Formicidae) foram classificadas como protetores contra herbivoria (BENTLEY, 1977; PREZOTO et al., 2016), sendo registrado a ocorrência no agroecossistema ou a não ocorrência do gênero/morfotipo. Para outros visitantes foi realizado somente o registro de ocorrência e não ocorrência, outros parâmetros não foram classificados. 


\section{Índices e análise dos dados}

Para realizar as análises os dados foram categorizados conforme descrito nas seções anteriores e foram realizadas inicialmente análises descritivas. Posteriormente, para avaliar a proporção entre as diferentes categorias de visitantes florais nos agroecossistemas foi empregado o teste exato de Fischer (LAW et al., 2001). Em seguida testou-se a distribuição dos visitantes florais nos agroecossistemas se eram semelhantes pelo teste de Qui-quadrado. Para avaliar a associação entre a variáveis estudadas realizou-se a correlação de postos de Spearmean. Para auxiliar nas análises utilizou-se a planilha eletrônica Excel 365 e o software livre $R$ (R CORE TEAM, 2017).

\section{RESULTADOS E DISCUSSÃO}

\section{Indicadores de sustentabilidade em agroecossistemas de urucuzeiro}

A média obtida nos quinze agroecossistemas para a qualidade do solo foi de 9,1. Sendo o maior valor de 10 no agroecossistema G, e o menor de 7,2 no agroecossistema D. O valor da média obtida para a saúde dos cultivos nos quinze agroecossistemas foi de 7,7, com o maior valor de 8,7 no agroecossistema 0 , e o menor valor de 6,1 no agroecossistema D. Os indicadores apontaram que a qualidade do solo e a saúde dos cultivos nos agroecossistemas de urucuzeiro é elevada por apresentarem valores próximos ao valor máximo (Figura 2).

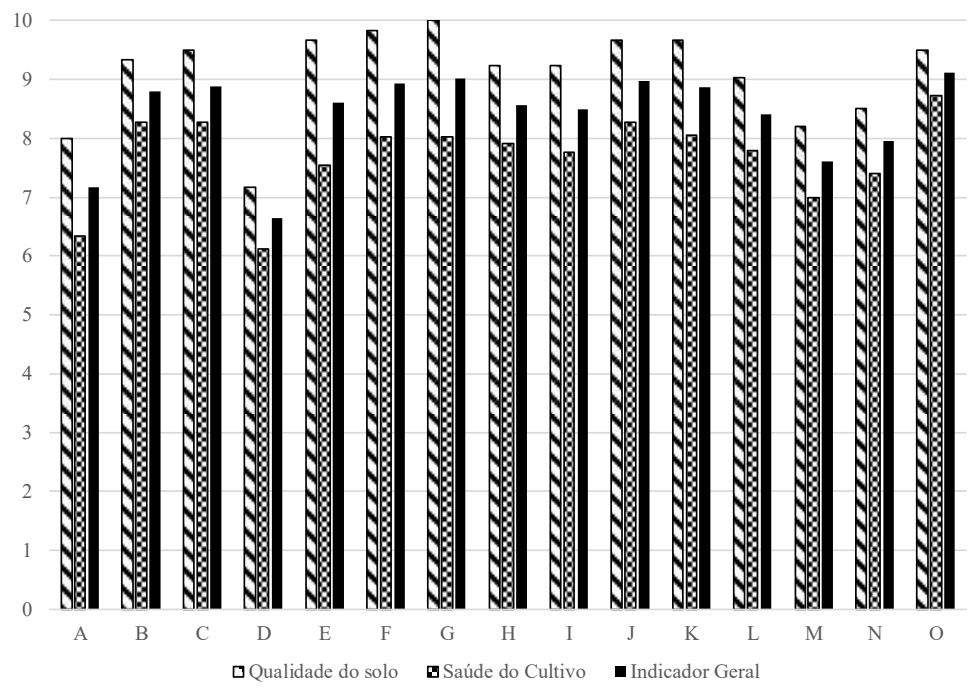

Figura 2: Valores médios dos indicadores de sustentabilidade de qualidade do solo e saúde dos cultivos obtidos em 15 agroecossistemas de urucuzeiro nos municípios de Seringueiras e São Miguel do Guaporé, cujas coletas foram realizadas em 2018, Amazônia Ocidental, Brasil.

Verificou-se que os agroecossistemas estudados apresentaram valores superiores a cinco para os indicadores de qualidade do solo e saúde dos cultivos, o que representa níveis acima da média. Isso demonstram sistemas produtivos sustentáveis para a região Vale do Guaporé. Quanto mais próximo do valor dez, mais qualidade e conservação apresenta-se o sistema em relação ao solo e sanidade do cultivo (ALTIERI et al., 2002; NICHOLLS et al., 2004). A interação entre os indicadores para a qualidade do solo e saúde do cultivo, permite visualizar o estado geral da cultura, considerando que quanto mais aproximar o índice ao diâmetro do círculo (valor 10), mais sustentável é o sistema (ALTIERI et al., 2002). 
Outros estudos foram realizados com a utilização dos mesmos indicadores de sustentabilidade, e os resultados mostram relações e interações entre os atributos para a qualidade do solo e saúde dos cultivos (FERREIRA, 2005; GUIMARÃES et al., 2013; SOUZA et al., 2016). E que os indicadores de saúde dos cultivos se relacionam com a diversidade funcional e com os elementos da agrobiodiversidade (MACHADO et al., 2006; CUNHA et al., 2014).

Sistemas de manejo com alta diversidade, com baixa entrada de insumos externos, e com diversidade de habitats no entorno, composto de diferentes plantas espontâneas, vegetação circundante à área de cultivo é beneficiado pelos serviços ecossistêmicos e exibi um maior nível de sustentabilidade (NICHOLLS et al., 2004). Ambientes diversificados é fundamental para melhorar a capacidade de respostas às adversidades, e tornar os cultivos mais resilientes, alcançando a sustentabilidade (HENAO, 2013; ALTIERI et al., 2015; NICHOLLS et al., 2015).

\section{Visitantes florais do urucuzeiro, ocorrência e comportamento de forrageamento}

$\mathrm{Na}$ coleta ativa foram capturadas 39 espécies (morfotipos) de abelhas, sendo 16 espécies (morfotipos) de abelhas de maior tamanho corporal e 23 espécies (morfotipos) de menor tamanho corporal, sendo que as de pequeno porte, 18 apresentavam capacidade vibratória e 5 delas não apresentavam comportamento vibratório ao coletar o pólen. Os polinizadores efetivos e ocasionais ocorreram em todos os agroecossistemas estudados (Tabela 1).

Os polinizadores efetivos (P.E) de urucuzeiro (Bixa orellana L.) são representados por abelhas de grande porte, tais como: Oxaea sp., Xylocopa sp., Bombus sp., Eulaema sp., Centris sp., Eufriesea sp. e Epicharis sp., o tempo de visita em cada flor varia de dois a seis segundos, dependendo da espécie. São espécies de abelhas que possuem grande tamanho corporal, representadas por indivíduos com mais de 14 $\mathrm{mm}$ de comprimento e $6 \mathrm{~mm}$ de largura (VIANA; KLEINERT, 2005). Estudos realizados destacam as abelhas como potenciais polinizadores, atuando como principais visitantes florais de Bixa orellana L., sendo as abelhas de grande porte os polinizadores efetivos (ALMEIDA et al., 1992; COSTA, 2005; MESQUITA, 2008; BONFIM et al., 2015; ROCHA et al., 2017).

Não foram encontradas diferenças entres as proporções das categorias de grupos funcionais de polinizadores efetivos e ocasionas ( $p$-valor $=0,6069$ ), bem como entre os grupos com vibração e sem vibração ( $p$-valor $=0,8856)$ nos agroecossistemas de urucuzeiro. Os polinizadores efetivos foram mais ocorrentes nos agroecossistemas N (17,9\%), B (15,4\%), L (15,4\%) e M (15,4\%). E menos ocorrentes nos agroecossistemas H $(2,6 \%)$ e I $(2,6 \%)$.

Sobre esse aspecto, os resultados indicaram redundância na distribuição dos polinizadores. As abelhas compreendem organismos funcionalmente equivalentes ou redundantes em suas funções, tanto que o desaparecimento de algumas dessas espécies não ocasiona perdas funcionais mensuráveis na comunidade (PAULA, 2013). Por outro lado, algumas espécies de abelhas ou guildas-chave são componentes biológicos significativos em um determinado ambiente, cuja presença ou ausência pode alterar drasticamente a estrutura e dinâmica dos ecossistemas (FENSTER et al., 2004; OLLERTON et al., 2011). 
Tabela 1: Ocorrência de visitantes florais da ordem Hymenoptera (abelhas) coletados em agroecossistemas de urucuzeiro no município de São Miguel do Guaporé e Seringueiras/RO, entre os meses de março e maio de 2018 . O comportamento das abelhas foi separado em: com vibração (C.V) e sem vibração (S.V), os grupos funcionais foram classificados em: polinizador efetivo (P.E) e polinizador ocasional (P.O).

\begin{tabular}{|l|l|l|l|l|}
\hline \multirow{2}{*}{ Agroecossistemas } & P.E & P.O & \multirow{2}{*}{ Total } \\
\cline { 2 - 4 } & C.V & C.V & S.V & \\
\hline A & 5 & 5 & 3 & 13 \\
\hline B & 6 & 4 & 1 & 11 \\
\hline C & 5 & 2 & 1 & 8 \\
\hline D & 5 & 5 & 3 & 13 \\
\hline E & 5 & 6 & 4 & 15 \\
\hline F & 3 & 4 & 4 & 11 \\
\hline G & 5 & 4 & 4 & 13 \\
\hline I & 1 & 5 & 2 & 8 \\
\hline J & 1 & 5 & 2 & 8 \\
\hline K & 3 & 5 & 3 & 11 \\
\hline L & 2 & 4 & 3 & 9 \\
\hline M & 6 & 7 & 3 & 16 \\
\hline N & 6 & 3 & 3 & 12 \\
\hline O & 7 & 5 & 1 & 13 \\
\hline
\end{tabular}

Foram observados visitando as flores do urucuzeiro um grupo diverso de Artrópodes, da classe Arachnida e da classe Insecta, sendo a ordem Hymenoptera a mais visualizada e presente nos agroecossistemas. As abelhas das famílias Andrenidae, Apidae e Halictidae, foram as mais ocorrentes nas áreas de estudo, observadas coletando principalmente o pólen como recurso floral (Tabela 2). Outros himenópteros também foram visualizados visitando as flores, sendo formigas (Formicidae) e vespas (Vespidae).

A Apis mellifera teve ocorrência em 82,35\% dos agroecossistemas estudados, são espécies de abelhas exóticas que se adaptam bem em habitats modificados. A ocorrência das abelhas de maior tamanho corporal tais como, Oxaea sp. $(76,47 \%)$, Xylocopa sp. $(70,59 \%)$ e Bombus sp. $(35,29 \%)$ foi significativa, visto que são espécies que preferem ambientes mais preservados.

As abelhas de menor tamanho corporal (inferior a $14 \mathrm{~mm}$ de comprimento e $6 \mathrm{~mm}$ de largura) (VIANA et al., 2005) foram enquadradas como polinizadores ocasionais, sendo representadas pelas abelhas de pequeno porte Apis mellifera, Scaptotrigona sp., Tetragonisca sp., Trigona sp., Melipona sp., Euglossa sp., Exomalopsis sp., Augochloropsis sp., são abelhas que pousam diretamente nos estames, andam muito sobre estes, permanecem mais tempo nas flores, contudo, na maioria das visitas não havia contato corporal com o estigma e, eventualmente, a polinização pôde ocorrer quando elas encostam o corpo no estigma, ou o pólen é liberado.

Nas observações, verificou-se a presença de abelhas durante todo o dia, com início das atividades nos primeiros raios de sol (07:00 h) e um declínio progressivamente após as 14:00 h, encerrando por completo após as 16:30 h, porém concentrou-se nos horários entre 8:00 h às 14:00 $h$, o período de maior visitação e forrageamento das abelhas nas flores de urucuzeiro. O período da manhã foi utilizado como o de maior frequência de visitação de polinizadores e visitantes florais (ALMEIDA et al., 1992; COSTA, 2005; COSTA et al., 2008; MESQUITA, 2008; BONFIM et al., 2015). 
Tabela 2: Ocorrência de visitantes florais da ordem Hymenoptera (abelhas) coletados em agroecossistemas de urucuzeiro, entre os meses de março e maio de 2018.

\begin{tabular}{|c|c|}
\hline Morfotipo & \% dos agroecossistemas \\
\hline Apis mellifera & 82,35 \\
\hline Melipona sp.1 & 76,47 \\
\hline Oxaea sp. & 76,47 \\
\hline Melipona sp.2 & 70,59 \\
\hline Trigona sp. 2 & 70,59 \\
\hline Xylocopa sp. & 70,59 \\
\hline Scaptotrigona sp. & 64,71 \\
\hline Exomalopsis sp.1 & 58,82 \\
\hline Augochloropsis sp. 2 & 47,06 \\
\hline Bombus sp. & 35,29 \\
\hline Eulaema sp.1 & 29,41 \\
\hline Centris sp.1 & 23,53 \\
\hline Centris sp. 2 & 17,65 \\
\hline Centris sp.4 & 17,65 \\
\hline Centris sp.7 & 17,65 \\
\hline Centris sp.9 & 17,65 \\
\hline Eufriesea sp. & 17,65 \\
\hline Eulaema sp.2 & 17,65 \\
\hline Augochloropsis sp.1 & 11,76 \\
\hline Augochloropsis sp.3 & 11,76 \\
\hline Euglossa sp.3 & 11,76 \\
\hline Exomalopsis sp.2 & 11,76 \\
\hline Exomalopsis sp.3 & 11,76 \\
\hline Melipona sp.3 & 11,76 \\
\hline Melipona sp.4 & 11,76 \\
\hline Tetragonisca sp. & 11,76 \\
\hline Augochloropsis sp.4 & 5,88 \\
\hline Augochloropsis sp.5 & 5,88 \\
\hline Centris sp.3 & 5,88 \\
\hline Centris sp.5 & 5,88 \\
\hline Centris sp. 6 & 5,88 \\
\hline Centris sp.8 & 5,88 \\
\hline Epicharis sp. & 5,88 \\
\hline Euglossa sp.1 & 5,88 \\
\hline Euglossa sp. 2 & 5,88 \\
\hline Euglossa sp.4 & 5,88 \\
\hline Melipona sp.5 & 5,88 \\
\hline Melipona sp. 6 & 5,88 \\
\hline Trigona sp.1 & 5,88 \\
\hline
\end{tabular}

Outros visitantes da ordem Hymenoptera foram visualizados visitando as flores de urucuzeiro, sendo formigas (Formicidae) e vespas (Vespidae), no entanto, só de passagem, pois estes estavam associados aos nectários extraflorais (NEFs) localizados na base da corola da flor e dos botões florais, que costuma estar relacionado com a atração e recompensa para artrópodes, que ao usar a secreção açucarada, protegem a região ao redor da flor de alguns insetos filófagos, que podem causar alguns danos a planta, e as formigas quando incomodadas possuem comportamento agressivo (BENTLEY, 1977), mas os visitantes dos NEFs não realizavam a polinização.

Verificou-se também a presença de insetos de outras ordens, entre eles, diferentes espécies de moscas, mosquitos e pernilongos (Diptera), grilos e gafanhotos (Orthoptera), besouros e vaquinhas (Coleoptera), percevejos e cigarras (Hemiptera), baratas (Blattodea), lagartas, borboletas e mariposas (Lepidoptera), tesourinhas (Dermaptera), louva-a-deus (Mantodea), bichos-lixeiros (Neuroptera) e libélulas (Odonata). 
$\mathrm{Na}$ coleta passiva com o uso de armadilhas de interceptação de voo, a ordem Hymenoptera apresentou uma riqueza (S) de 51 morfotipos, com abundância (N) de 737 indivíduos, distribuídos nas famílias Apidae, Halictidae, Formicidae e Vespidae. O índice de diversidade de Shannon-Wiener $\left(H^{\prime}\right)$ foi de 2,820 e o índice de equabilidade de Pielou (J) foi de 0,717. Os valores apresentados por agroecossistema estão na tabela 3.

A família Formicidae foi a única capturada na coleta passiva em todos os cultivos. No urucuzeiro as formigas estão associadas aos nectários extraflorais (BENTLEY, 1977), fazem parte de um grupo que desempenham importantes funções nos processos ecológicos, tais como proteção de plantas contra herbívoros, ciclagem de nutrientes, predação, herbivoria, estruturação física e química do solo, dispersão de sementes (VAZ-DE-MELO et al., 2009; BOLICO et al., 2012), constituindo um importante grupo na pirâmide de fluxo de energia.

Tabela 3: Riqueza (S), Abundância (N), Índice de Diversidade Shannon-Wiener ( $\left.\mathrm{H}^{\prime}\right)$ e Equabilidade de Pielou ( $\left.\mathrm{J}^{\prime}\right)$, da ordem Hymenoptera coletados com o uso de armadilhas em Agroecossistemas de urucuzeiro.

\begin{tabular}{|l|l|l|l|l|}
\hline Agroecossistemas & S & N & $H^{\prime}$ & J \\
\hline A & 9 & 118 & 0,86 & 0,39 \\
\hline B & 17 & 51 & 2,45 & 0,87 \\
\hline C & 7 & 16 & 1,72 & 0,88 \\
\hline E & 10 & 42 & 1,87 & 0,81 \\
\hline F & 11 & 90 & 1,86 & 0,77 \\
\hline G & 11 & 76 & 1,40 & 0,59 \\
\hline H & 6 & 19 & 1,51 & 0,84 \\
\hline J & 5 & 17 & 0,87 & 0,54 \\
\hline K & 12 & 47 & 2,02 & 0,81 \\
\hline L & 11 & 73 & 1,38 & 0,57 \\
\hline M & 11 & 29 & 1,55 & 0,65 \\
\hline N & 7 & 13 & 1,78 & 0,91 \\
\hline O & 8 & 10 & 2,03 & 0,97 \\
\hline
\end{tabular}

As vespas sociais coletadas apresentaram uma riqueza de 15 morfotipos, e abundância de 184 indivíduos, com distribuição em 11 agroecossistemas, este resultado já era esperado, pois as vespas estavam presentes dentro dos cultivos em busca de néctar extrafloral, e a armadilha utilizada na coleta é similar ao tipo de armadilha utilizada em estudos faunísticos de levantamento de vespas sociais (SOUZA et al., 2006; CLEMENTE, 2009; JACQUES et al., 2012; KLEIN et al., 2015; MACIEL, 2017). Diversos trabalhos têm demonstrado a importância das vespas sociais (Hymenoptera - Vespidae) dentro dos agroecossistemas, como inimigos naturais de diversas pragas e atuando como agentes no controle biológico em diversas culturas (CARPENTER et al., 2001; ELISEl et al., 2010; PREZOTO et al., 2016), além de algumas espécies estar relacionadas a polinização (HERMES et al., 2006; CLEMENTE et al., 2012), e representar bioindicadores de qualidade ambiental (SOUZA et al., 2010).

As abelhas (Apidae, Halictidae) são importantes visitantes florais do urucuzeiro (ALMEIDA et al., 1992; COSTA, 2005; COSTA et al., 2008; MESQUITA, 2008; BONFIM et al., 2015; ROCHA et al., 2017), fornecendo serviços de polinização para os cultivos. O baixo número de espécies coletados pode estar 
relacionado ao método de coleta, visto que estas espécies estavam presentes dentro dos cultivos, realizando visitações às flores.

\section{Relação entre a sustentabilidade e os visitantes florais}

Foi observada uma relação inversamente proporcional entre a riqueza nos grupos funcionais e o indicador geral de probabilidade (Tabela 4). O mesmo comportamento foi observado entre as os polinizadores ocasionais com comportamento vibratório. Entre os componentes do indicador de sustentabilidade, somente a saúde do cultivo teve relações inversamente proporcionais com a riqueza total nos grupos de polinizadores e os polinizadores ocasionais com comportamento vibratório.

Apesar de não ter sido evidenciada uma relação direta entre os grupos, essa variação pode estar relacionada com a paisagem no entorno das áreas estudadas. A riqueza de visitantes florais pode estar associada à proximidade do local em estudo com remanescentes florestais, a fragmentação dos habitats tem efeitos diretos na diversidade e abundância de várias espécies de abelhas polinizadoras (ELTZ et al., 2003; HARRIS et al., 2004; LENTINI et al., 2012). Várias características como tamanho, isolamento, proporção de vegetação nativa, qualidade, distância geográfica, e paisagem circundante é conhecida por influenciar a abundância e a frequência de visitas dos polinizadores (TSCHARNTKE et al., 2002). Em cultivos com menor proporção de vegetação nativa no entorno e menor número de fragmentos de habitats naturais, notou-se um declínio na riqueza e abundância dos polinizadores (TSCHARNTKE et al., 2002; RICKETTS et al., 2008; CARVALHEIRO et al., 2010; KLEIN et al., 2012; VIANA et al., 2012). Contudo, não foram encontradas associações dos polinizadores efetivos com nenhum dos componentes do indicador de sustentabilidade, nem com o indicador geral de sustentabilidade (Tabela 4).

Tabela 4: Teste de correlação de Spearman entre os indicadores Qualidade do Solo, Saúde do Cultivo, Indicador Geral, e riqueza dos grupos funcionais de polinizadores coletados em agroecossistemas de urucuzeiro. Os grupos funcionais foram classificados em: polinizador efetivo (P.E) e polinizador ocasional (P.O), sendo o ocasional subdividido em com vibração (C.V) e sem vibração (S.V).

\begin{tabular}{|c|c|c|c|c|c|c|}
\hline \multirow[t]{2}{*}{ Indicadores } & \multirow[t]{2}{*}{ Estatísticas } & \multicolumn{5}{|l|}{ Grupos } \\
\hline & & Total & P.E & P.O & P.O-C.V & P.O-S.V \\
\hline \multirow[t]{2}{*}{ Saúde do Cultivo } & $\rho$ & $-0,638$ & $-0,335$ & $-0,432$ & $-0,538$ & 0,443 \\
\hline & $\mathrm{p}$-valor & 0,011 & 0,223 & 0,108 & 0,038 & 0,098 \\
\hline \multirow[t]{2}{*}{ Qualidade do solo } & $\rho$ & $-0,226$ & $-0,382$ & 0,109 & $-0,277$ & $-0,135$ \\
\hline & $\mathrm{p}$-valor & 0,417 & 0,160 & 0,700 & 0,317 & 0,632 \\
\hline \multirow[t]{2}{*}{ Indicador Geral } & $\rho$ & $-0,508$ & $-0,418$ & $-0,216$ & $-0,495$ & 0,196 \\
\hline & $\mathrm{p}$-valor & 0,053 & 0,121 & 0,439 & 0,060 & 0,485 \\
\hline
\end{tabular}

Foi observado através do teste de correlação de Spearman que a riqueza, diversidade e abundância de visitantes florais não manteve relação direta com os indicadores de sustentabilidade, uma vez que o indicador geral (Tabela 5), na medida em que os valores dos indicadores se aproximavam ao valor máximo de sustentabilidade observado para os agroecossistemas, a riqueza, a diversidade e abundância não tinham a mesma relação, apresentando valores menores. Indicando que uma variável biológica, como a fauna de himenópteros, analisada em conjunto com atributos do solo e saúde dos cultivos, pode mostrar uma relação com outros fatores, podendo ser influenciada por outros componentes da paisagem, dentro e no entorno 
dos cultivos. O que torna o desenho e a composição dos agroecossistemas e de seu entorno determinantes na estruturação da comunidade de visitantes florais e potenciais polinizadores.

Tabela 5: Teste de correlação de Spearman dos indicadores Qualidade do Solo, Saúde do Cultivo, Indicador Geral, Riqueza (S), Abundância (N), Índice de Diversidade Shannon-Wiener ( $\left.H^{\prime}\right)$ e Equabilidade de Pielou (J'), da ordem Hymenoptera coletados com o uso de armadilhas de interceptação de voo em Agroecossistemas de urucuzeiro.

\begin{tabular}{|l|l|l|l|l|l|}
\hline \multicolumn{2}{|l|}{ Indicadores de sustentabilidade } & \multicolumn{4}{l|}{ Indicadores dos visitantes florais } \\
\cline { 3 - 6 } \multicolumn{2}{|l|}{} & $\mathrm{S}$ & $\mathrm{N}$ & $\mathrm{H}^{\prime}$ & $\mathrm{J}$ \\
\hline \multirow{2}{*}{ Saúde do Cultivo } & $\rho$ & 0,145 & $-0,095$ & $-0,197$ & $-0,047$ \\
\cline { 2 - 6 } & $\mathrm{p}$-valor & 0,605 & 0,736 & 0,481 & 0,869 \\
\hline \multirow{2}{*}{ Qualidade do solo } & $\rho$ & 0,061 & 0,104 & $-0,339$ & $-0,165$ \\
\cline { 2 - 6 } & $\mathrm{p}$-valor & 0,828 & 0,712 & 0,216 & 0,556 \\
\hline \multirow{2}{*}{ Indicador Geral } & $\rho$ & 0,049 & $-0,011$ & 0,355 & 0,570 \\
\cline { 2 - 6 } & $\mathrm{p}$-valor & 0,861 & 0,970 & 0,194 & $-0,160$ \\
\hline
\end{tabular}

A proximidade da vegetação nativa aos cultivos possibilita uma maior riqueza e mais frequência de visitas de polinizadores do que em cultivos mais distantes dos fragmentos naturais (BENEVIDES et al., 2009; KLEIN et al., 2012; TSCHARNTKE et al., 2012). Pesquisas corroboram que a fragmentação e a perda dos habitats naturais advindos da modificação da paisagem próximos aos cultivos podem ser prejudiciais para os polinizadores, principalmente abelhas (KREMEN et al., 2002; KREMEN et al., 2004; STEFFAN-DEWENTER et al., 2002; STEFFAN-DEWENTER et al., 2006; LARSEN et al., 2005; CANE et al., 2006; KREMEN et al., 2007; STEFFAN-DEWENTER et al., 2008).

Para permitir o estabelecimento de polinizadores próximos de cultivos, deve-se considerar, diferentes guildas de abelhas, isso porque grande parte das espécies de abelhas e outros visitantes florais, utilizam os fragmentos de vegetação nativa para construir seus ninhos e explorar muitos recursos. A influência de fragmentos de vegetação nativa, com habitats e paisagens diversificadas, são ambientes propícios ao estabelecimento e conservação dos polinizadores, e têm sido relatados por vários autores como importantes para garantir a polinização (KEARNS et al., 1998; STEFFAN-DEWENTER et al., 2002; KREMEN et al., 2004; KLEIN et al., 2007; KREMEN et al., 2007). Assim sendo conservar os habitats naturais próximos aos cultivos ajuda a manter os serviços de polinização (YAMAMOTO et al., 2010).

Outra provável causa para o declínio dos polinizadores e serviços de polinização, é o uso de agroquímicos (herbicidas e inseticidas) (KEARNS et al., 1998; KLEIN et al., 2007; POTTS et al., 2010; NAKASU et al., 2014). Seus resíduos ficam nas flores e contaminam o néctar e o pólen (RORTAIS et al., 2005) e, consequentemente, coloca em risco colônias e populações de abelhas e outros visitantes de matas próximas que visitam o local ou que polinizam as áreas de produção. Os efeitos de toxicidade estão relacionados às concentrações administradas, ao grau de exposição e a toxicidade do produto, podendo o efeito ser letal ou sub-letal (FREITAS et al., 2010; PINHEIRO et al., 2010).

A conservação dos serviços de polinização e atração dos polinizadores para as áreas de cultivo podem ser empregadas com várias práticas agrícolas amigáveis aos polinizadores. As principais foram descritas por vários autores, visando aumento na densidade, riqueza e diversidade de polinizadores nativos nas áreas agrícolas, além de promover a manutenção destes, que tenha local e oportunidades de nidificação, recursos 
florais, conectividade de habitat e redução de agrotóxicos (IMPERATRIZ-FONSECA, 2004; KLEIN et al., 2007; MAIA et al., 2012; GOULSON et al., 2015).

\section{CONCLUSÕES}

Os indicadores apontaram que a qualidade do solo e a saúde dos cultivos nos agroecossistemas de urucuzeiro é sustentável por apresentarem valores próximos ao máximo. Entre os visitantes florais observados, os polinizadores efetivos de urucuzeiro (Bixa orellana L.) são representados por abelhas de grande porte, que possuem maior tamanho corpóreo, pertencentes ao gênero Oxaea sp., Xylocopa sp., Bombus sp., Eulaema sp., Centris sp., Eufriesea sp. e Epicharis sp.

A riqueza de polinizadores ocasionais com comportamento vibratório apresentou relação inversamente proporcional ao indicador saúde do cultivo e ao indicador de sustentabilidade geral. Por outro lado, a riqueza, diversidade e abundância, de himenópteros não mantem relação direta com os indicadores de sustentabilidade, na medida em que os indicadores se aproximavam ao valor máximo de sustentabilidade para os agroecossistemas.

\section{REFERÊNCIAS}

AGUILAR, R.; ASHWORTH, L.; GALETTO, L.; AIZEN, M. A.. Plant reproductive susceptibility to habitat fragmentation: review and synthesis through a meta-analysis. Ecology Letters, v.9, p.968-980, 2006.

ALHO, C. J. R.. Importância da biodiversidade para a saúde humana: uma perspectiva ecológica. Estudos Avançados, v.26, p.151-166, 2012.

ALMEIDA, E. C.; PINHEIRO, A. L.. Biologia floral e mecanismo de reprodução em urucuzeiro (Bixa orellana L.) I. tipo fruto verde piloso. In: REUNIÃO TÉCNICO CIENTÍFICA SOBRE O MELHORAMENTO GENÉTICO DO URUCUZEIRO, 1992, Belém, PA. Anais. Belém: EMBRAPA, v.1, p.72-81, 1992.

ALTIERI, M. A.; NICHOLLS, C. I.. Un método agroecológico rápido para la evaluación de la sostenibilidad de cafetales. Manejo Integrado de Plagas y Agroecologia, Costa Rica, v.64, p.17-24, 2002.

ALTIERI, M. A.; NICHOLLS, C. I.; HENAO, A.; LANA, M. A.. Agroecology and the design of climate change-resilient farming systems. Agronomy for sustainable development, v.35, n.3, p.869-890, 2015.

ALVARES, C. A.; STAPE, J. L.; SENTELHAS, P. C.; GONÇALVES, J. L. de M.; SPAROVEK, G.. Köppen's climate classification map for Brazil. Meteorologische Zeitschrift, v.22, n.6, p.711728, 2014.

BACCARO, F. B.. Chave para as principais subfamílias e gêneros de formigas (Hymenopteras: Formicidae). Instituto Nacional de Pesquisa da Amazônia - INPA, 2006.

BAUER, D. M.; WING, I. S.. Economic consequences of pollinator declines: A synthesis. Agricultural and Resource Economics Review, v.39, p.368-383, 2010.
BAWA, K. S.; BULLOCK, S. H.; PERRY, D. R.; COVILLE, R. E.; GRAYUM, M. H.. Reproductive biology of tropical lowland rain forest tree. II pollination systems. American Journal Botany, v.72, p.346-356, 1985.

BENEVIDES, C. R.; GAGLIANONE, M. C.; HOFFMANN, M.. Visitantes florais do maracujá-amarelo (Passiflora edulis $f$. flavicarpa Deg. Passifloraceae) em áreas de cultivo com diferentes proximidades a fragmentos florestais na região Norte Fluminense, RJ. Revista Brasileira de Entomologia, São Paulo, v.53, n.3, p.415-421, 2009.

BENTLEY, B. L.. The protective function of ants visiting the extrafloral nectaries of Bixa orellana (Bixaceae). The Journal of Ecology, v.65, n.1, p.27-38, 1977.

BOLICO, C. F.; OLIVEIRA, E. A.; GANTES, M. L.; DUMONT, L. F. C.; CARRASCO, D. S.; D'INCAO, F.. Mirmecofauna (Hymenoptera, Formicidae) de duas marismas do Estuário da Lagoa dos Patos, RS: diversidade, flutuação de abundância e similaridade como indicadores de conservação. EntomoBrasilis, Vassouras, v.5, n.1, p.11-20, 2012.

BONFIM, M. S.; SILVA, S. O.; ALMEIDA, I. R. R.; PINA, W. C.. Abelhas (Hymenoptera: Apoidea) visitantes das flores de urucum (Bixa orellana Linnaeus 1753) em Teixeira de Freitas, Bahia, Brasil. Scientia Plena, v.11, n.5, 2015.

CANE, J. H.; MINCKLEY, R.; ROULSTON, T.; KERVIN, L.; WILLIAMS, N. M.. Multiple response of desert bee guild (Hymenoptera: Apiformes) to urban habitat fragmentation. Ecological Application, v.16, p.632-644, 2006.

CARPENTER, J. M.; MARQUES, O. M.. Contribuição ao estudo dos vespídeos do Brasil. Publicações Digitais, Salvador, v.2, p.1-147, 2001. 
CARVALHEIRO, L. G.; SEYMOUR, C. L.; VELDTMAN, R.; NICOLSON, S. W.. Pollination services decline with distance from natural habitat even in biodiversity-rich areas. Journal of Applied Ecology, Oxford, v.47, p.810-820, 2010.

CLEMENT, C. R.; CRISTO-ARAÚJO, M.; D’EECKENBRUGGE, G. C.; ALVES PEREIRA, A.; PICANÇO-RODRIGUES, D.. Origin and domestication of native Amazonian crops. Diversity, v.2, n.1, p.72-106, 2010.

CLEMENTE, M. A.. Vespas sociais (Hymenoptera, Vespidae) do Parque Estadual do Ibitipoca-MG: estrutura, composição e visitação floral. Dissertação (Mestrado em Ciências Biológicas, Zoologia) - Universidade Federal de Juiz de Fora, Juiz de Fora, 2009.

CLEMENTE, M. A.; LANGE, D.; DEL-CLARO, K.; PREZOTO, F.; CAMPOS, N. R.; BARBOSA, B. C.. Flower-visiting social wasps and plants interaction: Network pattern and environmental complexity. Psyche, v.2012, p.1-10, 2012.

CORBET, S. A.; WILLIAMS, I. H.; OSBORNE, J. L.. Bees and the pollination of crops and wild flowers in the European Community. Bee world, v.72 n.2, p.47-59, 1991.

COSTA, A. J. C.. Abelhas (Hymenoptera: Apoidea) visitantes das flores de urucum (Bixa orellana L.), em Vitória da Conquista - BA. Dissertação (Mestrado) - Universidade do Sudoeste da Bahia, Vitória da Conquista, 2005.

COSTA, A. J. C.; GUIMARÃES-DIAS, F.; PÉREZ-MALUF, R.. Abelhas (Hymenoptera: Apoidea) visitantes das flores de urucum em Vitória da Conquista, BA. Ciência Rural, v.38, n.2, p.534-537, 2008.

COSTANZA, R.; D'ARGE, R.; GROOT, R.; FARBER, S.; GRASSO, M.; HANNON, B.; LIMBURG, K.; NAEEM, S.; O'NEILL, R. V.; PARUELO, J.; RASKIN, R. G.; SUTTON, P.;VAN DEN BELT, M.. The value of the world's ecosystem services and natural capital. Nature, v. 387, p.253-260. 1997.

CUNHA, J. A. D. S.; BARROS, R. F. M.; MHEL, H. U.; SILVA, P. R. R.. Atributos agroecológicos de solo e caracterização de predadores generalistas no cultivo de melancia nos tabuleiros litorâneos do Piauí, Brasil. Revista Brasileira de Agroecologia, v.9, n.1, p.269-281, 2014.

D’ÁVILA, M.; MARCHINI, L. C.. Polinização realizada por abelhas em culturas de importância econômica no Brasil. Boletim da Indústria Animal, v.62 n.1, p.79-90, 2005.

DAILY, G. C.. Nature's services: societal dependence on natural ecosystems. Island Press, Washington, 1997.

DÍAZ, S.; FARGIONE, J.; CHAPIN III, F. S.; TILMAN, D.. Biodiversity loss threatens human well-being. PLoS biology, v.4, n.8, 2006.

ELISEI, T.; NUNES, J. V.; RIBEIRO JUNIOR, C.; FERNANDES JUNIOR, A. J.; PREZOTO, F.. Uso da vespa social Polistes versicolor no controle de desfolhadores de eucalipto. Pesquisa Agropecuária Brasileira (Impressa), v.45, n.9, p.958-964, 2010.

ELTZ, T.; BRÜHL, C. A.; IMIYABIR, Z.; LINSENMAIR, K. E. . Nesting and nest trees of stingless bees (Apidae: Meliponini) in lowland dipterocarp forests in Sabah, Malaysia, with implications for forest management. Forest Ecology and Management, v.172, n.2, p.301-313, 2003.

FAO. Conservation and management of pollinators for sustainable agriculture - the international response. In: FREITAS, B. M.; PEREIRA, J. O. P.. Solitary bees: conservation, rearing and management for pollination. Fortaleza: Imprensa Universitária 2004. p.19-25.

FENSTER, C. B.; ARMBRUSTER, W. S; WILSON, P.; DUDASH, M. R.; THOMSON, J. D.. Pollinization syndromes and flora specialization. Annual Review of Ecology, Evolution, and Systematics, v.35, p.375-403, 2004.

FERREIRA, J. M. L.. Indicadores de qualidade do solo e de sustentabilidade em cafeeiros arborizados. Dissertação (Mestrado em Agroecossistemas) - Universidade Federal de Santa Catarina, Florianópolis, 2005.

FREITAS, B. M.; PINHEIRO, J. N.. Efeitos sub-letais dos pesticidas agrícolas e seus impactos no manejo de polinizadores dos agroecossistemas brasileiros. Oecologia australis, Rio de Janeiro, v.14, n.1, p.282-298, 2010.

GALLAI, N.; SALLES, J-M.; SETTELE, J.; VAISSIÈRE, B. E.. Economic valuation of the vulnerability of world agriculture confronted with pollinator decline. Ecological Economics, v.68, p.810-821, 2009.

GIANNINI, T. C.; CORDEIRO, G. D.; FREITAS, B. M.; SARAIVA, A. M.; IMPERATRIZ-FONSECA, V. L.. The Dependence of crops for pollinators and the economic value of pollination in Brazil. Journal of economic entomology, v.108, n.3, p.849857, 2015.

GOULSON, D.; NICHOLLS, E.; BOTÍAS, C.; ROTHERAY, E. L.. Bee declines driven by combined stress from parasites, pesticides, and lack of flowers. Science, v.347, n.6229, p.1255957. 2015.

GROOT, R. S.; WILSON, M. A.; BOUMANS, R. M. J.. A typology for the classification, description and valuation of ecosystem functions, goods and services. Ecological Economics, v.41, n.3, p.393-408, 2002.

GUIMARÃES, G. P.; MENDONÇA, E. S; PASSOS, R. R.; ANDRADE, F. V.; MACHADO, R. V.. Avaliação da qualidade do solo e cafeeiros em propriedade familiar do Território do Caparaó-ES. Revista Brasileira de Agroecologia, v.8, n.3, p.236-246, 2013

HARRIS, L. F.; JOHNSON, S. D.. The consequences of habitat fragmentation for plant-pollinator mutualisms. International Journal of Tropical Insect Science, v.24, n.1, p.29-43, 2004.

HENAO, A.. Propuesta metodológica de medición de la resiliencia agroecológica en sistemas socio-ecológicos: un estudio de caso en los andes colombianos. Agroecología, v.8, n.1, p.85-91, 2013.

HERMES, M. G.; KÖHLER, A.. The flower-visiting social wasps (Hymenoptera, Vespidae, Polistinae) in two areas of Rio Grande do Sul State, Southern Brazil. Revista Brasileira de Entomologia, v.50, n.2, p.268-274, 2006. 
IBGE. Instituto Brasileiro de Geografia e Estatística. Manual técnico da vegetação brasileira. 2 ed. Rio de Janeiro: IBGE, 2012.

IBGE. Instituto Brasileiro de Geografia e Estatística. Manual técnico de pedologia. Diretoria de Geociências, Coordenação de Recursos Naturais e Estudos Ambientais, Manuais Técnicos em Geociências. 3 ed. Rio de Janeiro: IBGE, 2015.

IMPERATRIZ-FONSECA, V. L.. Serviços aos ecossistemas, com ênfase nos polinizadores e polinização. São Paulo: USP, 2004.

JACQUES, G. C.; CASTRO, A. A.; SOUZA, G. K.; SILVA-FILHO, R.; SOUZA, M. M.; ZANUNCIO, J. C.. Diversity of Social Wasps in the Campus of the Universidade Federal de Viçosa in Viçosa, Minas Gerais State, Brazil. Sociobiology, v.59, n.3, p.1053-1063, 2012.

KEARNS, C. A.; INOUYE, D. W.; WASER, N. M.. Endangered mutualisms: The Conservation of Plant-Pollinator Interactions. Annual Review of Ecology and Systematics, v.29, p.83-112, 1998.

KEVAN, P. G.; VIANA, B. F.. The global decline of pollination services. Biodiversity Journal of Life on Earth, v.4, n.4, p.0308. 2003.

KEVAN, P. G.; PHILLIPS, T. P.. The economics impacts of pollinator declines: an approach to assessing the consequences. Conservation Ecology, v.5, n.1, 2001.

KLEIN, A. M.; VAISSIERE, B. E.; CANE, J. H.; STEFFANDEWENTER, I.; CUNNINGHAM, S. A.; KREMEN, C.; TSCHARNTKE, T.. Importance of pollinators in changing landscapes for world crops. Proceedings of the Royal Society of London B, v.274, n.1608, p.303-313, 2006.

KLEIN, A. M.; BRITTAIN, C.; HENDRIX, S. D.; THORP, R.; WILLIAMS, N.; KREMEN, C.. Wild pollination services to California almond rely on semi-natural habitat. Journal of Applied Ecology, Oxford, v.49, p.723-732, 2012.

KLEIN, A. M.; VAISSIÈRE, B. E.; CANE, J. H.; STEFFANDEWENTER, I.; CUNNINGHAM, S. A.; KREMEN, C.; TSCHARNTKE, T.. Importance of pollinators in changing landscapes for world crops. Proceedings of the Royal Society B, v.274, p.303-313, 2007.

KLEIN, R. P.; SOMAVILLA, A.; KÖHLER, A.; CADEMARTORI, C. V.; FORNECK, E. D.. Space-time variation in the composition, richness and abundance of social wasps (Hymenoptera: Vespidae: Polistinae) in a forest-agriculture mosaic in Rio Grande do Sul, Brazil. Acta Scientiarum. Biological Sciences, v.37, n.3, p.327-335, 2015.

KREMEN, C.; WILLIAMS, N. M.; AIZEN, M. A.; GEMMILHERREN, B.; LEBUHN, G.; MINCKLEY, R.; PACKER, L.; POTTS, S. G.; ROULSTON, T.; STEFFAN-DEWENTER, I.; VÁZQUEZ, P.; WINFREE, R.; ADAMS, L.; CRONE, E. E.; GREENLEAF, S. S.; KEIT, T. H.; KLEIN, A. M.; REGETZ, J.; RICKETTS, T. H.. Pollination and other ecosystem services produced by mobile organisms: a conceptual framework for the effects of land-use change. Ecology letters, v.10, p.299-314, 2007.
KREMEN, C.; WILLIAMS, N. M.; BUGG, R. L.; FAY, J. P.; THORP, R. W.. The area requirements of an ecosystem service: crop pollination by native bee communities in California. Ecology Letters, v.7, p.1109-1119, 2004.

KREMEN, C.; WILLIAMS, N. M.; THORP, R. W.. Crop pollination from native bees at risk from agricultural intensification. Proceedings of the National Academy of Sciences of the United States of America, v.99, n.26, p.16812-16816, 2002.

LARSEN, T. H.; WILLIAMS, N. M.; KREMEN, C.. Extinction order and altered community structure rapidly disrupt ecosystem functioning. Ecology Letters, v.8, p.538-547, 2005.

LAW, G. R.; COX, D. R.; MACHONOCHIE, N. E. S.; SIMPSON, J.; ROMAN, E.; CARPENTER, L. M.. Large tables. Biostatistics, v.2, n.2, p.163-171. 2001

LENTINI, P. E.; MARTIN, T. G.; GIBBONS, P.; FISCHER, J.; CUNNINGHAM, S. A.. Supporting wild pollinators in a temperate agricultural landscape: Maintaining mosaics of natural features and production. Biological Conservation, v.149, n.1, p.84-92, 2012.

LIMA FILHO, J. A.; MORAIS, T. B.; FREIRE, O. O.; LEITE FILHO, F. G.. Aula prática utilizando um novo modelo de armadilha com garrafa PET na captura de insetos em uma área de caatinga em Princesa Isabel, PB. In: CONGRESSO NORTE NORDESTE DE PESQUISA E INOVAÇÃO, 8. Anais. Salvador: CONNEPI, 2013.

LIMA FILHO, J. A.; OLIVEIRA, A. G. C.; FREIRE, O. O.; BEZERRA, B. M. G.; SANTOS, V. M.. Captura de Insetos Utilizando um Novo Modelo de Armadilha com Garrafa PET em uma Área do IFPB Campus Campina Grande, PB. Gaia Scientia, v.8, n.1, p.74-79, 2014.

LORENZI, H.; MATOS, F. J.. Plantas medicinais no Brasil: nativas e exóticas cultivadas. Nova Odessa: Instituto plantarum, 2002.

MACHADO, C. D. T.; VIDAL, M. C.. Avaliação participativa do manejo de agroecossistemas e capacitação em agroecologia utilizando indicadores de sustentabilidade de determinação rápida e fácil. Planaltina: Embrapa Cerrados, 2006.

MACIEL, T. T.. Coleta de vespas sociais em estudos de diversidade: estado da arte e otimização do método de armadilhas atrativas. Dissertação (Mestrado em Ciências Biológicas) - Universidade Federal de Juiz de Fora, Juiz de Fora, 2017.

MAIA, A. C. N.; KHAN, A. S.; OLIVEIRA, J. A. D.; FREITAS, B. M.; LIMA, P. V. P. S.. Adoção de práticas amigáveis aos polinizadores na caju cultura Nordestina. In: CONGRESSO DA SOCIEDADE BRASILEIRA DE ECONOMIA, ADMINISTRAÇÃO E SOCIOLOGIA RURAL-SOBER, 50. Anais. 2012.

MESQUITA, F. L. A.. visitantes das flores do urucuzeiro (Bixa orellana L.) e suas eficiências de polinização. Dissertação (Mestrado) - Universidade Federal do Ceará: Fortaleza, 2008.

NAKASU, E. Y. T.; WILLIAMSON, S. M.; EDWARDS, M. G.; FITCHES, E. C.; GATEHOUSE, J. A.; WRIGHT, G. A.; 
GATEHOUSE, A. M. R.. Novel biopesticide based on a spider venom peptide shows no adverse effects on honeybees. Proceedings of the Royal Society B, v.281, n.20140619, 2014.

NICHOLLS, C. I.; ALTIERI, M. A.; DEZANET, A.; LANA, M.; FEISTAUER, D.; OURIQUES, M.. A rapid, farmer-friendly agroecological method to estimate soil quality and crop health in vineyard systems. Biodynamics, Pottstow, v.20, n.5, p.33-44, 2004

NICHOLLS, C. I.; HENAO, A.; ALTIERI, M. A.. Agroecología y el diseño de sistemas agrícolas resilientes al cambio climático. Agroecología, v.10, n.1, p.7-31, 2015.

OLLERTON, J.; WINFREE, R.; TARRANT, S.. How many flowering plants are pollinated by animals?. Oikos, v.120, n.3, p.321-326, 2011

PAULA, G. A. R.. Perspectiva histórica e estudo de conceitos em ecologia funcional. Oecologia Australis, v.17, n.3, p.331346, 2013.

PINHEIRO, J. N.; FREITAS, B. M.. Efeitos letais dos pesticidas agrícolas sobre polinizadores e perspectivas de manejo para os agroecossistemas brasileiros. Oecologia Australis, Rio de Janeiro, v.14, n.1, p.266-281, 2010.

POTTS, S. G.; BIESMEIJER, J. C.; KREMEN, C.; NEUMANN, P.; SCHWEIGER, O.; KUNIN, W. E.. Global pollinator declines: trends, impacts and drivers. Trends in Ecology \& Evolution, v.25, n.6, p.345-353, 2010.

PREZOTO, F.; BARBOSA, B. C.; MACIEL, T. T.; DETONI, M. Agroecossistemas e o serviço ecológico dos insetos na sustentabilidade. In: RESENDE, L. O.; PREZOTO, F.; BARBOSA, B. C.; GONÇALVES, E. L.. Sustentabilidade: Tópicos da Zona da Mata Mineira. Juiz de Fora, Real Consultoria em Negócios Ltda, 2016. p.19-30.

R CORE TEAM. R: A language and environment for statistical computing. R Foundation for Statistical Computing, Vienna, Austria. 2017.

RICKETTS, T. H.; REGETZ, J.; STEFFAN-DEWENTER, I.; CUNNINGHAM, S. A.; KREMEN, C.; BOGDANSKI, A.; GEMMILL-HERREN, B.; GREENLEAF, S. S.; KLEIN, A. M.; MAYFIELD, M. M.; MORANDIN, L. A.; OCHIENG, A.; POTTS, S. G.; VIANA, B. F.. Landscape effects on crop pollination services: are there general patterns?. Ecology Letters, v.11, p.499-515, 2008.

ROCHA, A. N.; POLATTO, L. P.. Bixa orellana L. (Bixaceae): dependência de polinizadores e estratégias de forrageio dos visitantes florais. Biota Amazônia, v.7, n.3, p.1-7, 2017.

RONDÔNIA. Secretaria de Estado do Desenvolvimento Ambiental (SEDAM). Boletim Climatológico de Rondônia Ano 2010. COGEO - SEDAM / Coordenadoria de Geociências. Secretaria de Estado do Desenvolvimento Ambiental. Porto Velho: COGEO - SEDAM, 2012.

RORTAIS, A.; ARNOLD, G.; HALM, M-P.; TOUFFET-BRIENS, F.. Modes of honeybees exposure to systemic insecticides: estimated amounts of contaminated pollen and nectar consumed by different categories of bees. Apidologie, v.36, p.71-83, 2005.
SANDIFER, P. A.; SUTTON-GRIER, A. E.; WARD, B. P.. Exploring connections among nature, biodiversity, ecosystem services, and human health and well-being: Opportunities to enhance health and biodiversity conservation. Ecosystem Services, v.12, p.1-15, 2015.

SANDY-CUEN, P. M.; BECERRA, R.. Manejo campesino de recursos naturales. El achiote. BioDiversitas, Belo Horizonte, v.7, p.7-11, 2003.

SANTOS, H. G.; CARVALHO JÚNIOR, W.; DART, R. O.; ÁGLIO, M. L. D.; SOUSA, J. S.; PARES, J. G.; FONTANA, A.; MARTINS, A. L. S.; OLIVEIRA, A. P.. O novo mapa de solos do Brasil: legenda atualizada. Rio de Janeiro: Embrapa Solos, 2011.

SANTOS, R. F.; PIEDADE KIILL, L. H.; PINHEIRO ARAÚJO, J. L.. Levantamento da flora melífera de interesse apícola no município de Petrolina-PE. Revista Caatinga, v.19, n.3, p.221-227, 2006

SILVEIRA, F. A.; MELO, G. A. R.; ALMEIDA, E. A. B.. Abelhas brasileiras: sistemática e identificação. Belo Horizonte, 2002.

SOUZA, H. A.; FARIAS, J. L. S.; PAIVA, F. E. P.; GUEDES, F. L.; POMPEU, R. C. F. F.; ROWS, J. R. C.. Avaliação participativa de indicadores de solo e sanidade de cultivos em sistemas de produção em comunidade rural no semiárido brasileiro. Revista Brasileira de Agroecologia, v.11, n.3, p.206-215, 2016.

SOUZA, M. M.; LOUZADA, J.; SERRÁO, J. E.; ZANUNCIO, J. C. Social wasps (Hymenoptera: Vespidae) as indicators of conservation degree of riparian forests in Southeast Brazil. Sociobiology, v.56, n.2, p.387-396, 2010.

SOUZA, M. M.; PREZOTO, F.. Diversity of social wasps (Hymenoptera: Vespidae) in semideciduous forest and cerrado (Savanna) regions in Brazil. Sociobiology, v.47, n.1, p.135-147, 2006.

STEFFAN-DEWENTER, I.; KLEIN, A. M.; ALFERT, T.; GAEBELE, V.; TSCHARNTKE, T.. Bee diversity and plant-pollinator interactions in fragmented landscapes.. In: WASER, N. M.; OLLERTON, J.. Specialization and generalization in plantpollinator interactions. Chicago: Chicago Press, 2006. p.387408.

STEFFAN-DEWENTER, I.; MÜNZENBERG, U.; BÜRGER, C.; THIES, C.; TSCHARNTKE, T.. Scale-dependent effects of landscape structure on three pollinator guilds. Ecology, v.83, n.5, p.1421-1432, 2002.

STEFFAN-DEWENTER, I.; WESTPHAL, C.. The interplay of pollinator diversity, pollination services and landscape change. Journal of Applied Ecology, v.45, p.737-741, 2008.

TSCHARNTKE, T.; STEFFAN-DEWENTER, I.; KRUESS, A.; THIES, C.. Characteristics of insect populations on habitat fragments: A mini review. Ecological Research, v.17, p.229239, 2002.

TSCHARNTKE, T.; TYLIANAKIS, J. M.; RAND, T. A.; DIDHAM, R. K.; FAHRIG, L.; BATÁRY, P.; BENGTSSON, J.; CLOUGH, Y.; CRIST, T. O.; DORMANN, C. F.; EWERS, R. M.; FRÜND, J.; HOLT, R. D.; HOLZSCHUH, A.; KLEIN, A. M.; KLEIJN, D.; KREMEN, C.; LANDIS, D. A.; LAURANCE, W.; LINDENMAYER, 
D.; SCHERBER, C.; SODHI, N.; STEFFAN-DEWENTER, I.; THIES, C.; VAN DER PUTTEN, W. H.; WESTPHAL, C.. Landscape moderation of biodiversity patterns and processes - eight hypotheses. Biological Reviews, v.87, p.661-685, 2012.

VAZ-DE-MELO, F.; BROWN, G.G.; CONSTANTINO, R.; LOUZADA, J. N. C.; LUIZÃO, F. J.; MORAIS, J. W.; ZANETTI, R.. A importância da meso e macrofauna do solo na fertilidade e como bioindicadores. Boletim Informativo da SBCS, v.34, p.38-43, 2009.

VIANA, B. F.; BOSCOLO, D.; MARIANO NETO, E.; LOPES, L. E.; LOPES, A. V.; FERREIRA, P. A.; PIGOZZO, C. M.; PRIMO, L. M.. How well do we understand landscape effects on pollinators and pollination services?. Journal of Pollination Ecology, v.7, n.5, p.31-41, 2012.

VIANA, B. F.; KLEINERT, A. D. M. P.. A community of flowervisiting bees (Hymenoptera: Apoidea) in the coastal sand dunes of northeastern Brazil. Biota neotropica, v.5, n.2, p.113,2005

YAMAMOTO, M.; SILVA, C. I.; AUGUSTO, S. C.; BARBOSA, A. A. A.; OLIVEIRA, P. E.. The role of bee diversity in pollination and fruit set of yellow passion fruit (Passiflora edulis forma flavicarpa, Passifloraceae) crop in Central Brazil. Apidologie, v.43, p.515-526, 2012.

YAMAMOTO, M.; BARBOSA, A. A. A.; OLIVEIRA, P. E. A. M.. A polinização em cultivos agrícolas e a conservação das áreas naturais: o caso do maracujá-amarelo (Passiflora edulis $f$.. flavicarpa Deneger). Oecologia Australis, v.14, n.1, p.174192, 2010.

ZHANG, W.; RICKETTS, T. H.; KREMEN, C.; CARNEY, K. M.; SWINTON, S. M.. Ecosystem services and dis-services to agriculture. Ecol. Econ., v.64, p.253-260, 2007. 